\title{
Biology of muscle atrophy and of its recovery by FES in aging and mobility impairments: roots and by-products
}

Ugo Carraro (1,2), Helmut Kern (3,4), Paolo Gava (2), Christian Hofer (4), Stefan Loefler (4), Paolo Gargiulo (5,6), Simone Mosole (2,4), Sandra Zampieri (2,4), Valerio Gobbo (7), Barbara Ravara (2,4), Francesco Piccione (1), Andrea Marcante (1), Alfonc Baba (1), Sheila Schils (8), Amber Pond (9), Francesco Gava $(2,4)$

(1) IRRCS Fondazione Ospedale San Camillo, Venezia, Italy; (2) Laboratory of Translational Myology of the Interdepartmental Research Center of Myology, Department of Biomedical Science, University of Padova, Italy; (3) Institute of Physical Medicine and Rehabilitation, Wilhelminenspital, Vienna, Austria; (4) Ludwig Boltzmann Institute of Electrical Stimulation and Physical Rehabilitation, Vienna, Austria; (5) Institute for Biomedical and Neural Engineering, Reykjavík, Iceland; (6) Landspítali, Reykjavík, Iceland; (7) C.N.R. Institute of Neuroscience, Department of Biomedical Science, University of Padova, Italy; (8) EquiNew, 8139 900th Street, River Falls, WI, USA; (9) Anatomy Department, Southern Illinois University, School of Medicine, Carbondale, Illinois, USA

\begin{abstract}
There is something in our genome that dictates life expectancy and there is nothing that can be done to avoid this; indeed, there is not yet any record of a person who has cheated death. Our physical prowess can vacillate substantially in our lifetime according to our activity levels and nutritional status and we may fight aging, but we will inevitably lose. We have presented strong evidence that the atrophy which accompanies aging is to some extent caused by loss of innervation. We compared muscle biopsies of sedentary seniors to those of life long active seniors, and show that these groups indeed have a different distribution of muscle fiber diameter and fiber type. The senior sportsmen have many more slow fiber-type groupings than the sedentary people which provides strong evidence of denervation-reinnervation events in muscle fibers. It appears that activity maintains the motoneurons and the muscle fibers. Premature or accelerated aging of muscle may occur as the result of many chronic diseases. One extreme case is provided by irreversible damage of the Conus and Cauda Equina, a spinal cord injury (SCI) sequela in which the human leg muscles may be completely and permanently disconnected from the nervous system with the almost complete disappearance of muscle fibers within 3-5 years from SCI. In cases of this extreme example of muscle degeneration, we have used 2D Muscle Color CT to gather data supporting the idea that electrical stimulation of denervated muscles can retain and even regain muscle. We show here that, if people are compliant, atrophy can be reversed. A further example of activity-related muscle adaptation is provided by the fact that mitochondrial distribution and density are significantly changed by functional electrical stimulation in horse muscle biopsies relative to those not receiving treatment. All together, the data indicate that FES is a good way to modify behaviors of muscle fibers by increasing the contraction load per day. Indeed, it should be possible to defer the muscle decline that occurs in aging people and in those who have become unable to participate in physical activities. Thus, FES should be considered for use in rehabilitation centers, nursing facilities and in critical care units when patients are completely inactive even for short periods of time.
\end{abstract}

Key Words: Muscle power, master athletes, aging decay, muscle denervation/reinnervation, type groupings, long-term denervated muscles, h-b FES-induced muscle recovery, subsarcolemmal mitochondria, equine muscle spasm 
There is something in our genome that dictates life expectancy and nothing has yet been discovered which can stop this decline. To date there is no record of any immortal human being. It is a common experience that power produced by skeletal muscle decreases as we age. How this decline occurs and whether or not there are abrupt increases in the rate of decay at some point are debated questions.

\section{Lessons from Masters: 1. World record series of Master Athletes}

Professor AV Hill stated in a famous 1925 paper that information concerning the physiology and pathology of mobility may be found in the results of sport competitions. ${ }^{1}$ Indeed, the rate of muscle power deterioration that occurs with aging can be deduced from the decline noted in the world records of Master Athletes in various track and field events. Studies on this subject are numerous and our recent results, in line with those of others, ${ }^{2}$ produce a trend-line for the power decline which commences at the age of 30 and continues to decrease toward zero by the age of 110 years. ${ }^{3}$ What surprised us when Paolo Gava presented his charts (Fig. 1) is that muscle power declines in a linear fashion and that the loss of power is a rather consistent $25 \%$ every 20 years. ${ }^{3}$ Figure 1 shows that performance level declines with age almost linearly whether the activity be running, jumping or throwing. Note that at a fixed time (whichever you choose) each world champion is by definition the best person in his field of competition: just one out of five billion people today. Even these exceptional people, with the best genetic background, the best attitude to train and perform, with the best trainers, lose power drastically as they age. Thus, there is something in our genome that dictates this event and nothing (as yet) has been found which can prevent this decline. Of course, all the other five billion people have performance lines which fall under those of the Masters, but if they are healthy people, the trend-line will be the same: they will be at their best around 30 years and their performance will decline until death. Luckily, at seventy years we still have roughly $50 \%$ of our youth power, more than enough for a normal life style. Size and power of our muscles can go up and down several times in our lifetime according to what we do and with our nutritional and activity statuses; however, no matter how well we may fight aging, in the end, we will lose. One may maintain strong results for years, but we are all fighting against our genome-dictated life expectancy.

\section{Lessons from Masters: 2. Rate of denervation and reinnervation events}

Age-related changes occur even in the muscle of very active people, Master Athletes included. We have collected data which compares the muscle of young people with that of seniors (either sedentary people or those with a lifelong history of high activity level). The evidence strongly suggests that age-related decay is

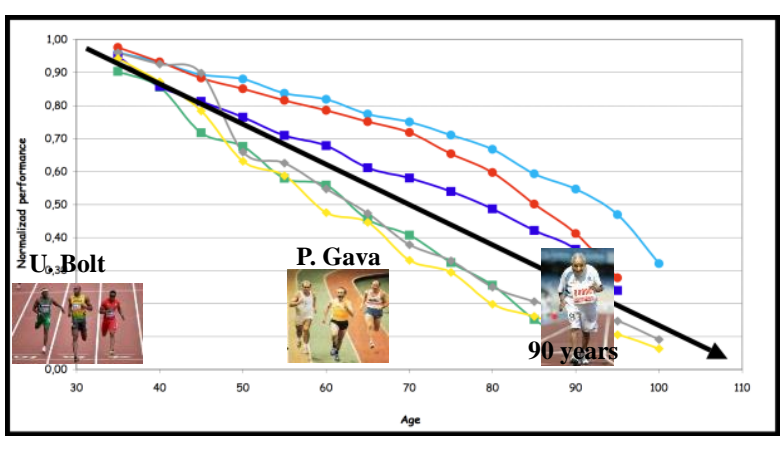

Age-related decline of skeletal muscle power in master athletes 2013 Spring Padua Muscle Days Paolo Gava, Padova, Italy

Fig 1. Age-related decline of skeletal muscle power derived from world records of running, jumping and throwing events of Masters of different age classes. Lines: Light blue, 100 meters run; Red, 400 meters run;Blue, Long Jump; Gray, High Jump; Yellow, Shot Put; Green, Hammer Throw. The insets show $U$ Bolt (around 30 years), $P$ Gava ( $<60$ years) and a 90 year old Japanese Master world record-holding athlete pictured the day they established the respective world record. Whatever the extent of training, even in the extreme cases of Master world record-holding men, muscle power almost linearly decreases with age pointing to around 110 years of human survival. The points of each individual line represent the normalized power of each World Record of the Master Athletes (seldom, the same athlete was able to hold the record for different age classes). The size changes of muscles and of myofibers do not fully explain the extent of dysfunction observed during aging. Though muscle disorders of ultrastructure and of molecular mechanisms may explain the additional functional decline, we are interested in studying if muscle fiber athrophy/apoptosis driven by denervation is a contributing factor (see Fig. 2).

influenced by loss of motoneurons and/or of motor axons. $^{2,4,5}$ In sedentary seniors, the vast majority of muscle fibers appears to co-express both slow and fast Myosin Heavy Chains (MHC) and some of these are of a peculiar small size and angulated appearance, likely being denervated muscle fibers. ${ }^{6}$ In contrast, Sandra Zampieri stresses the point that there are bigger muscle fibers in the senior sportsmen, ${ }^{6}$ but that there is also a larger number of slow type groupings in these muscles. ${ }^{4-6}$ This suggests that one fiber, which was fast and surrounded by slow ones, became a slow one. This is a "type grouping", the final evidence of "denervation" of muscle fibers, because they are "reinnervated" by a different motoneuron axon (Fig. 2 ). The slow type fibers and type-groupings are significantly increased in senior sportsmen relative to both the youth and sedentary seniors. We may, indeed, 

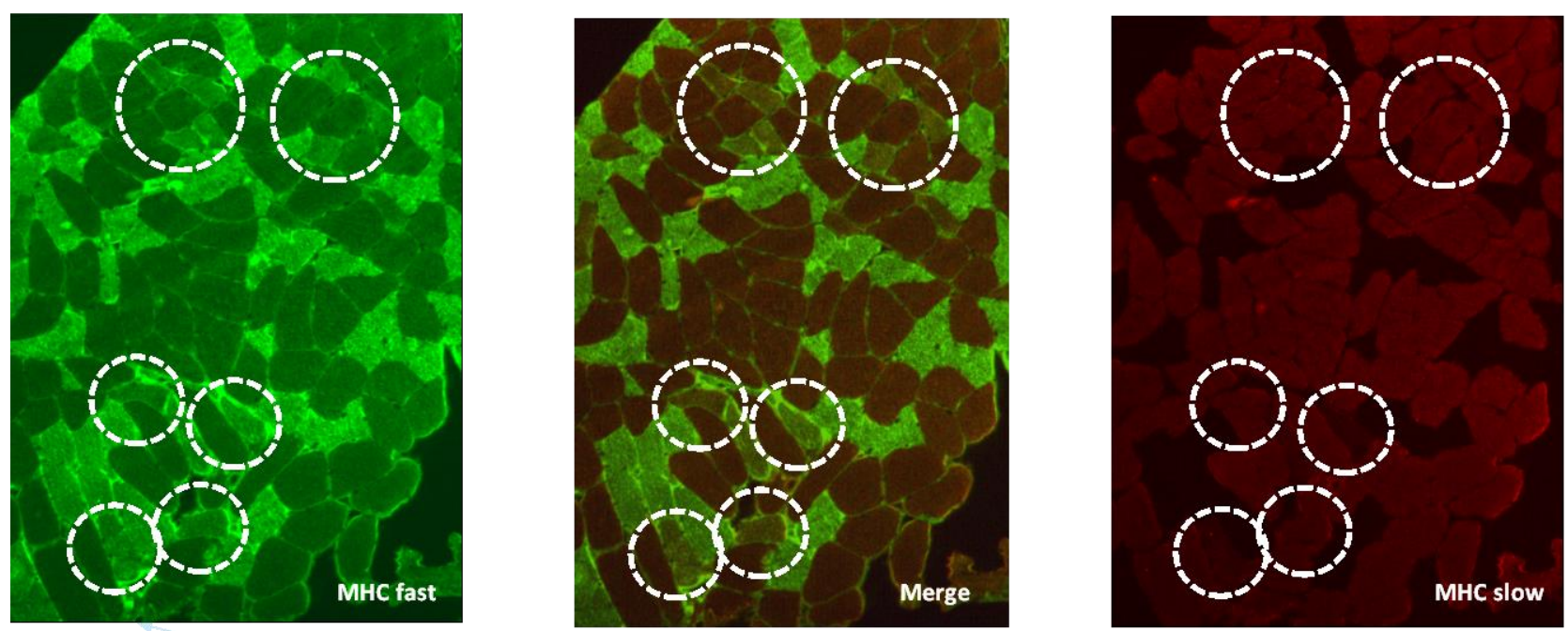

Fig 2. In muscle biopsies of lifelong highly active senior amateur sportsmen, the MHC co-expression in type-grouped slow muscle fibers suggests that activity-driven reinnervation by preferentially saved slow type motoneurons occurs. The MHC co-expressing myofibers are of normal size and some of these fill the gaps between clusters of slow myofibers (that is, slow type-groupings). We suggest these fibers were denervated fast muscle fibers preferentially reinnervated by axons sprouting from slow motoneurons.

observe that up to $90 \%$ of fibers in the muscle biopsies of senior sportsmen are slow type. This is an extreme example, but it means that the slow motoneurons may colonize as many fibers as possible, almost all of those available in an area. Further and more importantly, in the senior sportsmen, muscle fibers co-expressing both slow and fast myosin heavy chain proteins are normal in size and often fill the gaps which occur between clusters of slow myofibers. We suggest that these fibers are reinnervated by axons sprouting from slow motoneurons. ${ }^{5}$

Why the denervated muscle fibers are preferentially reinnervated by axons sprouting from slow motoneurons is a critical point in aging. Our opinion is that this is related to the fact that slow motoneurons are much more often activated per day than fast motoneurons, and that their frequent activation spares them. Thus, activity maintains the slow-type motoneurons and muscle fibers. It is possible that as much as $1 \%$ of fibers lose innervation every month. This may not seem like a great number, but over 40 years this $1 \%$ per month results in a substantial loss. Further, it may, at least in part, explain why older people have slower muscles. Regardless, the fact is that sedentary senior people and lifelong highly active seniors have a different distribution of muscle fiber diameter and type. The senior sportsmen who were highly active up to the day of the biopsies had been active for as much as 40 years. This is majorly different from doing sports for only 10 years during youth. The idea that denervation occurs naturally in aging is based on evidence of reinnervation, and we may say this because in a normal muscle of young sportsmen there are few to no type-groupings. ${ }^{7}$
We tried to correlate the type of lifelong sport activity of seniors with the extent of type groupings, but could not find any relationship. ${ }^{5}$ In our opinion, the key factor is the increased amount of activity, whether strength or endurance training. Our conclusion is that senior sportsmen have greater myofiber diameters, a lower percentage of denervated myofibers and a higher number of type groupings because they are doing more physical activity. It indicates that exercise has beneficial effects on age-related muscle degradation because it promotes muscle fiber reinnervation, saving preferentially the slow type motoneurons. The slow motoneurons are activated more often per day and, therefore, are more active than the fast ones. Activity maintains, thus, the motoneurons (and/or their axon sprouting potential) and the muscle fibers. ${ }^{5}$

\section{Premature muscle aging due to complete and permanent denervation of leg muscles}

Premature or accelerated aging may occur as the result of many chronic diseases, including those which involve loss of innervation.

One extreme case is irreversible Conus and Cauda Equina syndrome, a spinal cord injury (SCI) sequela in which the leg muscles could be completely and permanently disconnected from the nervous system and thus muscle fibers almost completely disappear within 3-5 years from the SCI. ${ }^{8-17}$ When a young man of 30 years, loses all of the connections from his spinal cord to the leg muscles, he will lose muscle contraction below the SCI lesion. Without contractions, muscle fibers will disappear within a few years, being replaced by connective tissues. 


\section{Biology of muscle atrophy and of its recovery by FES}

Eur J Transl Myol - Basic Appl Myol 2015; 25 (4): 221-230

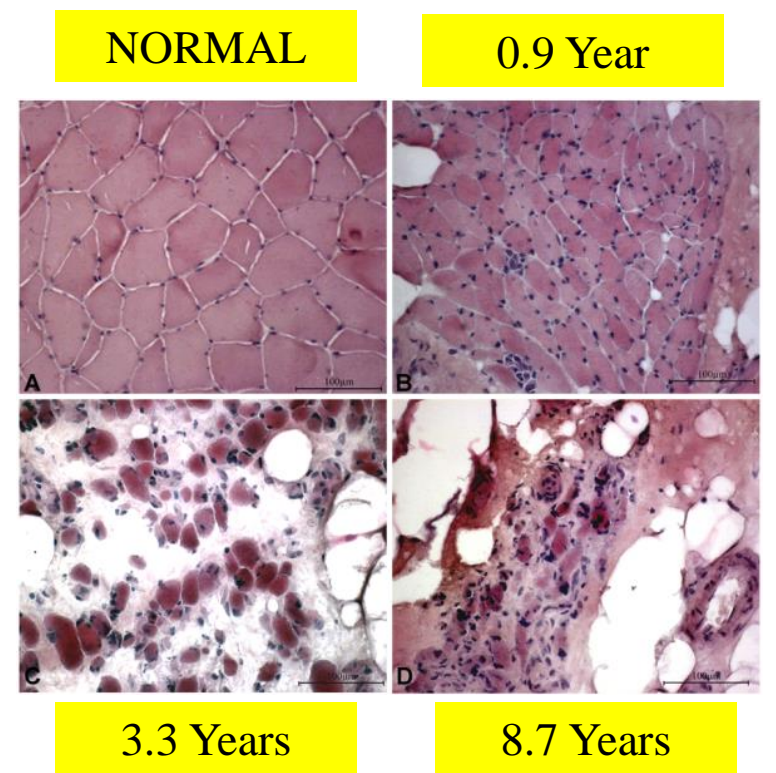

Fig 3. Permanent long-term denervation simulates premature aging in muscle. Human skeletal muscle undergoes four defined phases subsequent to permanent long-term denervation: 1) Loss of contractlity and ultrastructural disorganization (in months); 2) Atrophy (up to 2-years after SCI); 3) Severe atrophy (3 to 6 ys after SCI); 4) Loss of myofibers and muscle degeneration (> 3 years after SCI). These are the unexpected results of the EU Program RISE: Use of electrical stimulation to restore standing in paraplegics with long-term denervated degenerated muscles (Contract no. QLG5-CT2001-02191). ${ }^{14}$

Is there anything that can be done in this extreme situation to prevent these negative changes?

First we should look at the effects of long-term denervation on human muscle (Fig. 3). Four phases have been identified: 1) ultrastructural disorganization and loss of electrical stimulation-induced contractility (within months); 2) muscle atrophy (up to 2-years after $\mathrm{SCI})$; 3) muscle degeneration with severe muscle atrophy (3 to 6 years after SCI); and 4) loss of myofibers (> 3 years after $\mathrm{SCI}$ ). At some point after more than 10 years of permanent denervation, there are almost no more muscle fibers in the thighs of these patients. Obviously, the consequences of this degree of muscle loss can be devastating, resulting in increased morbidity and mortality in these patients (see for a recent review ${ }^{17}$ ). However, we have shown that home-based functional electrical stimulation (h-b FES) of permanently denervated muscles stops and reverts the degeneration of the skeletal muscle tissue. ${ }^{7-19}$

Figure 4 presents 2D Muscle Color Computer Tomography scans of thigh muscles in transverse section demonstrating the efficacy of h-b FES on long term permanently denervated leg muscles. Here, the relative amount of muscle increased in each patient after 2 years of h-b FES with a concomitant decrease in the abundance of interstitial tissues. Indeed, if people are compliant with the use of h-b FES, then atrophy of completely and irreversibly denervated leg muscles can be reversed. Despite the difficulties involved with muscle responding to electrical stimulation, there are ways to defer the onset of the late phases of muscle degeneration. In particular, if FES training is started earlier that 1 year after SCI the retention of muscle is much greater, almost complete. If one induces many contractions daily in those muscle fibers that are otherwise destined to die, they will survive and contract for up to 50 years, but only if one stimulates them with very long, high amplitude impulses delivered by large surface electrodes designed for stimulating denervated muscles, using the patterns of stimulation developed in Vienna by Prof. Helmut Kern, Prof. Winfried Mayr and collaborators. ${ }^{8-19}$

Now commercial devices, designed for electrical stimulation of long term permanently denervated muscles and capable of producing the needed patterns are available. Therefore, this excellent therapy should become available to more people for preservation of skeletal muscle. Please note in the panels of Fig. 4, B to $\mathrm{E}$ that over time from the point of SCI there is progressive loss of muscle tissue (in red) with an increase in interstitial tissue (yellow, green and blue represent fat, loose and fibrous connective tissues, respectively). After two additional years of permanent denervation, but with FES treatment (Fig. 4, G to J), the muscles became almost normal tissue.

The most impressive evidence for the positive effects of electrical stimulation of permanently denervated muscles is the extreme differences seen between panels $\mathrm{E}$ and $\mathrm{J}$ of Fig. 4. Even after tissue degeneration is prevalent (at 5.4 years from SCI), two more years of home based electrical stimulation substantially improved the muscle content (percentage of red area). Indeed, the colors represent different tissues, computed on the basis of their density (i.e., the amount of absorbed X ray, Hounsfield Units): they are not "freehand painted" onto the images. Thus, the results are actual and astonishing. Electrical stimulation can, thus, greatly improve even badly degenerated muscle tissue. One must ask why electrical stimulation is not more widely used and, in fact, is considered ineffective for treatment of permanently denervated muscles by some specialists in the medical field.

It is because the generic words "electrical stimulation" alone are meaningless. For FES to be effective, one has to know which kind of electrical stimulation pattern to use and how much and how often to administer it. That is, one must know how to correlate appropriate protocols with desired clinical effects. The case in Fig. 4 (panels $\mathrm{E}$ and $\mathrm{J}$ ) is very interesting: two years of 
Biology of muscle atrophy and of its recovery by FES

Eur J Transl Myol - Basic Appl Myol 2015; 25 (4): 221-230
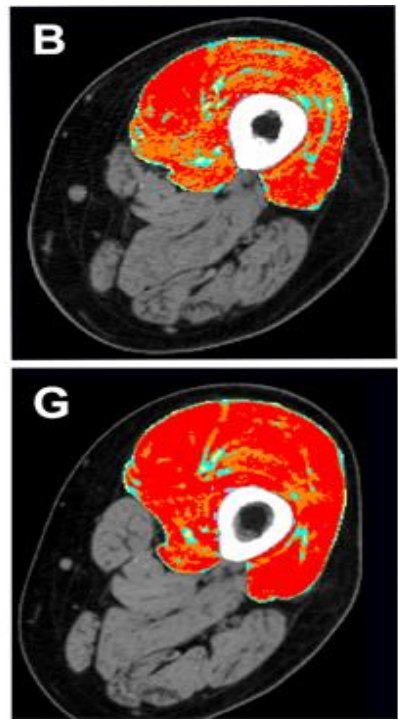
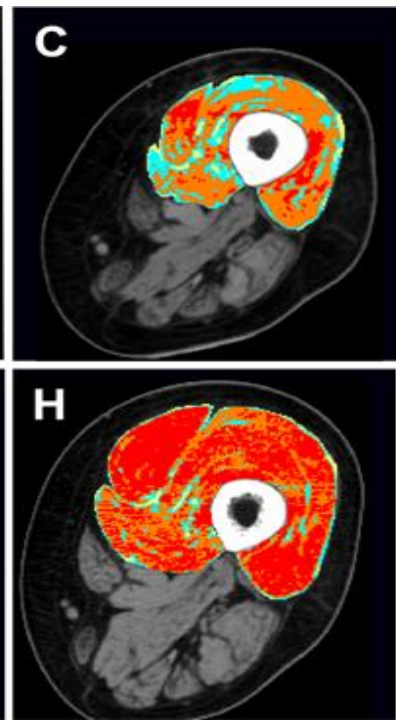
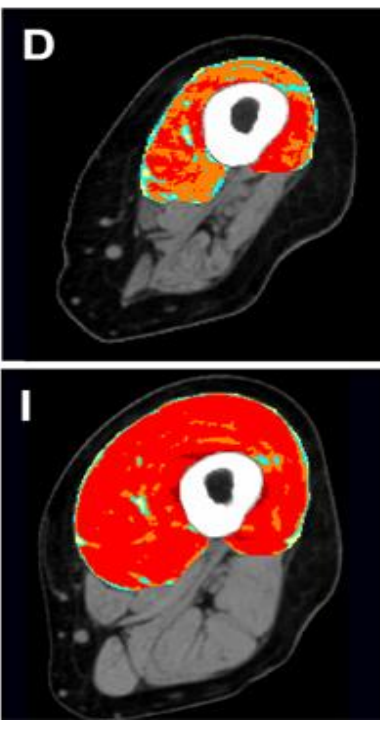
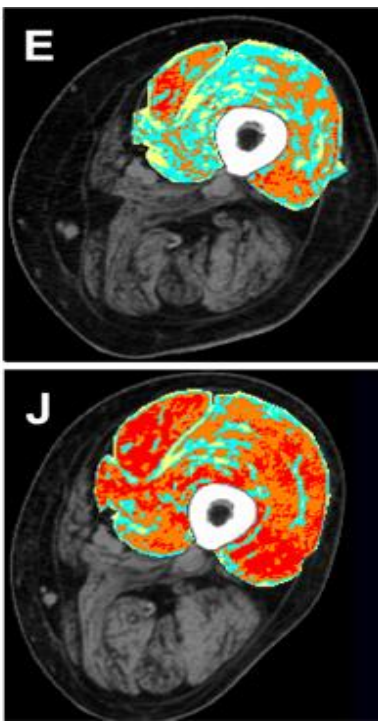

Fig 4. Recovery from permanent denervation (i.e., premature muscle aging) by h-b FES: the $2 D$ Color CT evidence. Color scans of thigh muscles before ( $B$ to $E)$ and after 2 years $(G$ to $J)$ of home-based functional electrical stimulation (h-b FES). Each panel shows that the cross-sectional area and the quality of quadriceps muscles in patients starting $h$ - $b$ FES at different time points after denervation $(B, 1.2 ; C, 1.7 ; D, 3.2 ; E$, 5.4 years) increased after 2 years of home training $(G, H, I, J$, respectively). Moreover, the interstitial tissues that increase with the denervation time (compare yellow, green, and blue areas in panels $B, C, D$, and $E$ ) decreased in the respective patient after 2 years of $h-b F E S(G, H, I, J$, respectively).

stimulation starting at 5 years after SCI vastly improved the content of muscle fibers as demonstrated by both muscle biopsy and computed tomography analyses. ${ }^{14}$ As further evidence of the efficacy of $h-b$ FES for recovery of permanently denervated skeletal muscles, Figure 5 shows that once FES is discontinued (for an additional 5 years), the recovered leg muscle deteriorates again. ${ }^{20}$ Figure 5 demonstrates that the previous muscle recovery was not due to reinnervation of the muscle fibers, but it was due to the trophic effect of the FESinduced contractions. If electrical stimulation is discontinued, the great effects seen with the treatment disappear. The conclusion is that h-b FES training (5 times a week at $3 \mathrm{~h}$ a day) reverts atrophy and maintains trophism of the muscle fibers in long-term denervated human muscles. ${ }^{9,15-17}$

The final report of the RISE trial was published in the best of the rehabilitation journals, i.e., Neurorehabilitation and Neural Repair (2010). ${ }^{14}$ Soon afterward, people familiar with our hard evidence ceased to criticize functional electrical stimulation. However, even now, if one talks with the vast majority of neurologists and physiatrists, who are not yet aware of our results, they say that denervated muscle cannot be maintained and certainly not regenerated - that it is simply not possible.

We need additional readily perceived evidence to convince skeptics that FES (even of permanently denervated muscle tissue) is effective.

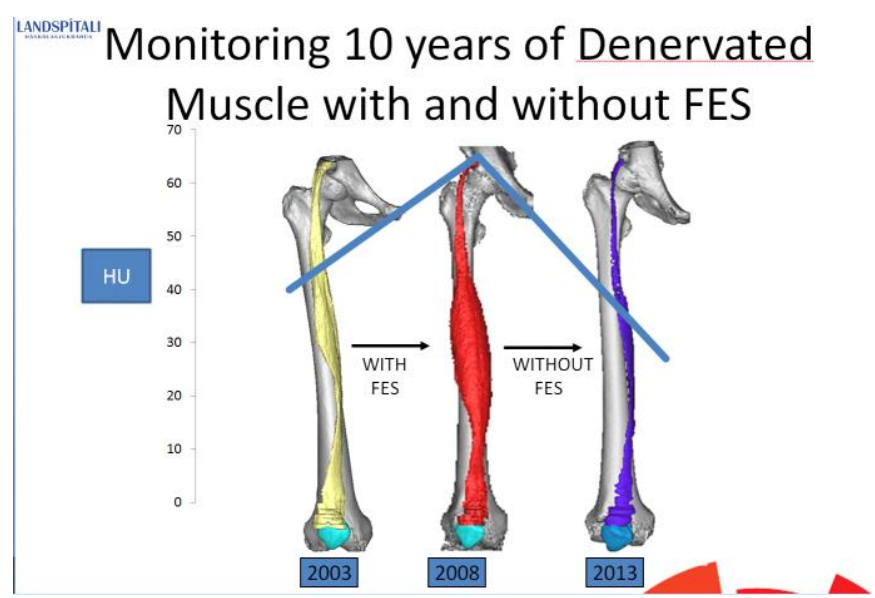

Fig 5. 3 D Color Muscle CT reconstruction of the rectus femoris. Reversible $h-b$ FES-induced recovery The patient started $h-b$ FES in 2003 (depicted in yellow). After 5 years of FES the muscle increased in size and density (2008). After 5 additional years without $h-b$ FES the muscle appears, as would be expected, for a nonstimulated denervated muscles, i.e., even more atrophic and fibrotic (2013). The mean muscle density (expressed in Hounsfield Unit, HU) at different times from SCI are indicated by the ascending and descending light blue line. 
Biology of muscle atrophy and of its recovery by FES

Eur J Transl Myol - Basic Appl Myol 2015; 25 (4): 221-230
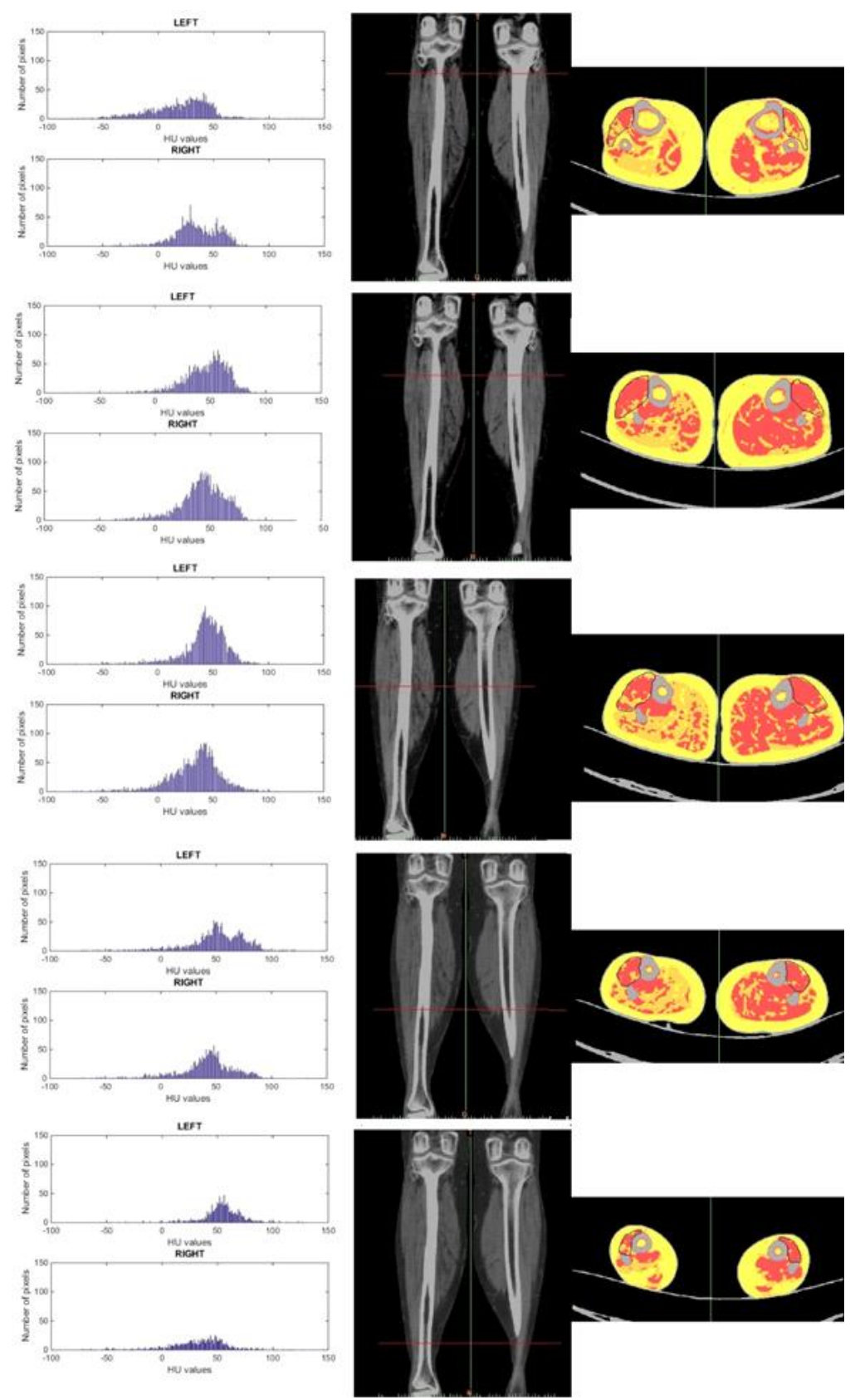

Fig 6. 2D muscle color CT scan. The distribution analysis of the ranges of Hounsfield Unit in the histograms (left panels) allows for much more detailed quantitation of the changes occurring within the soft tissues of the leg (i.e., fat in yellow, muscles in red or orange, according to their density). During inpatient 2 months rehabilitation, the subject was treated each day for 30 minutes with electrical stimulation for denervated muscles, bilaterally on the anterior and lateral side of the leg. Histograms of HU distribution show that the right leg contains the same amounts of subcutaneous fat as the left leg, but more intramuscular fat and low density muscle at the expense of the normal density muscle. Two months of conventional physiotherapy and electrical stimulation improved in the right leg by $+5 \%$ the content of low density muscle at the expenses of the intramuscular fat and fibrous-dense connective tissue (results not presented).

A by-product of our previous studies presented in Figure 6, clinical imaging by 2D and 3D Muscle Color Computed Tomography may be useful to collect final evidence of the FES effectiveness. The methodology allows us to follow and evaluate the effects of FES on an entire muscle more effectively because the analysis is sensitive enough to recognize $5 \%$ changes in muscle quality and volume, a functionally significant percent change when a person is near to exhausting the functional reserve. Widely used in cardiology 


\section{Biology of muscle atrophy and of its recovery by FES \\ Eur J Transl Myol - Basic Appl Myol 2015; 25 (4): 221-230}

imaging, ${ }^{21}$ the false color approach is ignored in clinical imaging of skeletal muscle tissue. We hope to have convinced the readers that its advantages offset the low risks of irradiation, in particular during follow-up of supervised trials, to add unbiased quantitative evidence to clinical assessments.

\section{A horse model to assess FES effectiveness by morphometry of subsarcolemmal mitochondria}

Finally, we would like to discuss a recent example of the effectiveness of FES for equine epaxial muscle spasm. Its relevance here is related to the fact that psychological factors are, reasonably, less important or absent in horse treatments. In this example, we explored the different types of mitochondria present in skeletal muscle fibers, either subsarcolemmal or intermyofibrillar and their differential response to needs and activation loads of muscle fibers. ${ }^{22-24}$

We had the opportunity to analyze 12 muscle biopsies (6 pre- and 6 post-FES) from 6 FES-treated horses. Previous preliminary histopathologic analyses suggested that stimulated muscles were more damaged after than before treatment.

Could this be the result of the electrical stimulation? Our additional more careful morphometric analyses exclude it. Indeed, only one horse presents with obvious evidence of post-FES muscle damage (foci of severe muscle fiber atrophy) whereas the other 5 horses, which had the same type and amount of electrical stimulation, display only scanty evidence of muscle atrophy (possibly resulting from denervation) in both pre- and post-stimulation biopsies. $^{23}$

We wish to stress here that any muscle damage detected in post-FES tissue analyses is too often immediately attributed to FES without further evaluation. However, if one does morphometry properly (with random sampling and statistical evaluation) the correlation between FES and muscle damage often disappears, as is the case in this group of horse muscle biopsies.

Indeed, heavy electrical stimulation, (that is, electroporation) is routinely used to deliver plasmid DNA to the muscle tissue of experimental rodents without reported tissue damage. ${ }^{25,26}$

Anyhow, in the post-FES horse biopsies NADH-TR staining of mitochondria (Fig. 7, A) shows an increase of those mitochondria that are localized near the sarcolemma (i.e., subsarcolemmal mitochondria, also known as paravascular mitochondria because they are usually in between muscle nuclei and the capillaries, Fig. $7 \mathrm{~B}) .^{23}$ It is interesting to note that, in recent observations comparing young and very old (30 months) rat muscles (EDL, soleus and diaphragm) subjected to neurectomy, ${ }^{24}$ we found that the abundance of subsarcolemmal mitochondria decreases with age and even more so after one week of denervation, whatever the animal age (manuscript in preparation). Feliciano Protasi and others have shown very interesting data concerning the structural relationships of mitochondria with triads in the intermyofibrillar spaces. ${ }^{6,9,11,14}$ They conclude that the physical interaction is part of the mechanism(s) that provide the needed amount of ATP to the contractile machinery. ${ }^{27}$

To what are the subsarcolemmal mitochondria providing ATP? This is an open question, since there are several different essential cytoplasmic and nuclear processes occurring with or without muscle contractions. Anyhow, in muscle fibers there are different types of mitochondria and they respond differently to various muscle fiber needs. We may only
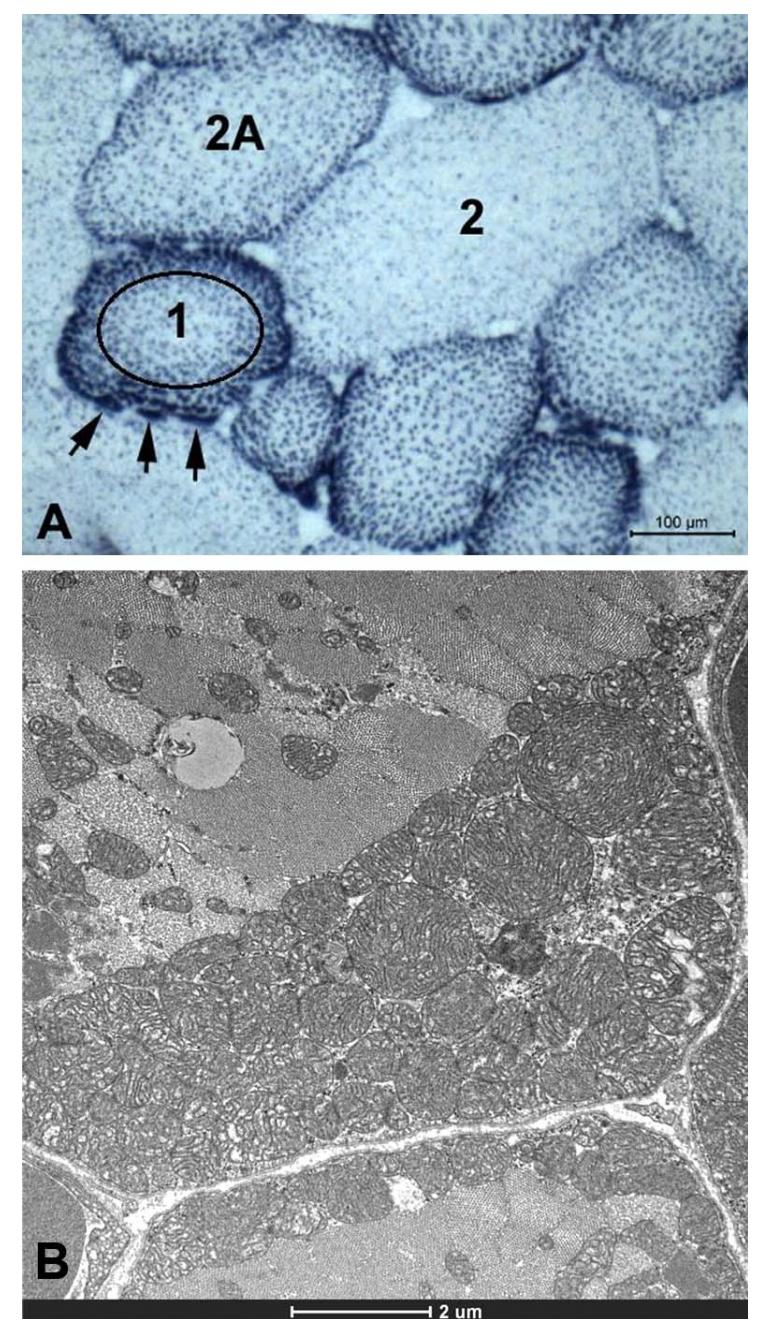

Fig 7. Mitochondrial evidence of FES effectiveness. A. NADH-TR stain. Type 2 large, glycolytic muscle fiber with low-density mitochondria, Type $2 A$ medium, glycolytic-oxidative muscle fiber, Type 1 small, oxidative muscle fiber with high-density mitochondria patches (black arrows). The circle defines the central intermyofibrillar area from the coronal subsarcolemmal high-density mitochondrial area. $B$. Electron microscopy of high density mitochondrial patches. Note that the patches are located between capillaries ( upper right and lower left corners), i.e., in paravascular location. 


\section{Biology of muscle atrophy and of its recovery by FES \\ Eur J Transl Myol - Basic Appl Myol 2015; 25 (4): 221-230}

say, for now, that mitochondrial distribution and density are significantly changed in horses post-FES muscle biopsies. This indicates that the clinical improvements observed in the horse are possibly related to increased muscle perfusion induced by FES stimulation. Likewise as in human cases, ${ }^{8-20}$ electrical stimulation provides clinically relevant results in the treatments of equine epaxial muscle spasm. It seems to be a good way to increase contraction per day, i. e., mimic volitional exercise and, thus, modify the behaviors of the muscle fibers.

\section{Conclusion and perspectives}

In summary, it appears that age-related decline in muscle power is partially attributable to loss of innervation and that this loss can be deferred by life-long high-level activity. ${ }^{28}$ Diseases involving permanent denervation show similar, but premature and much more severe muscle deterioration. We have shown that, with appropriate protocols, h-b FES can inhibit muscle degeneration and that it can also actually reverse it. ${ }^{29}$ It should be possible to stave off age-related muscle decline in aging people and others who have become unable to participate in physical activities. Thus, FES should be considered for use in rehabilitation centers, nursing facilities and in critical care units when patients are completely inactive even for short periods of time.

\section{Acknowledgement}

This work was supported by European Regional Development Fund - Cross Border Cooperation Programme Slovakia - Austria 2007-2013 (InterregIVa), project Mobilität im Alter, MOBIL, N_00033 (partners: Ludwig Boltzmann Institute of Electrical Stimulation and Physical Rehabilitation, Austria, Center for Medical Physics and Biomedical Engineering, Medical University of Vienna, Austria, and Faculty of Physical Education and Sports, Comenius University in Bratislava, Slovakia); Austrian national co-financing of the Austrian Federal Ministry of Science and Research; Ludwig Boltzmann Society (Vienna, Austria).

Some of the research reported in this publication was supported by the National Institute of Arthritis and Musculoskeletal and Skin Diseases of the National Institutes of Health under Award Number NIH NIAMS 1R03AR053706-01A2 to ALP. The content is solely the responsibility of the authors and does not necessarily represent the official views of the National Institutes of Health.

This paper is an edited transcription of the talk presented by Ugo Carraro to the Interreg IVa Final Meeting November 8 2014, Vienna, Austria.

\section{Corresponding Author}

Francesco Gava, Via Armistizio, 87, 35142, Padova, Italy. E-mail: francescospa@alice.it

\section{E-mail of coauthors}

Ugo Carraro: ugo.carraro@ospedalesancamillo.net Helmut Kern: wil.pys.kern-forschung@wienkav.at
Paolo Gava: Paologavastra@alice.it Christian Hofer: christian.hofer@wienkav.at Stefan Loefler: stefan.loefler@wienkav.at Paolo Gargiulo: paologar@landspitali.is Simone Mosole: simone.mosole@studenti.unipd.it Sandra Zampieri: sanzamp@unipd.it Valerio Gobbo: gobbov@bio.unipd.it Barbara Ravara: barbara.ravara@unipd.it Francesco Piccione:

francesco.piccione@ospedalesancamillo.net Andrea Marcante:

andrea.marcante@ospedalesancamillo.net Alfonc Baba: alfonc.baba@ospedalesancamillo.net Sheila Schils: sbschils@EquiNew.com Amber Pond: apond@siumed.edu

\section{References}

1. Hill AV. The physiological basis of athletic records. The Scientific Monthly 1925;2:409-428.

2. Mitchell WK, Williams J, Atherton P, Larvin M, Lund J, Narici M. Sarcopenia, dynapenia, and the impact of advancing age on human skeletal muscle size and strength; a quantitative review. Front Physiol 2012 Jul 11;3:260. doi: 10.3389/fphys.2012.00260. eCollection 2012.

3. Gava P, Kern H, Carraro U. Age-associated power decline from running, jumping, and throwing male masters world records. Exp Aging Res. 2015;41(2):115-35. doi: 10.1080/0361073X.2015.1001648.

4. Mosole S, Rossini K, Kern H, et al. Significant increase of vastus lateralis reinnervation in 70year sportsmen with a lifelong history of highlevel exercise. Eur J Transl Myol - Basic Appl Myol 2013;23:117-22.

5. Mosole S, Carraro U, Kern H, et al. Long-term high-level exercise promotes muscle reinnervation with age. J Neuropathol Exp Neurol 2014;73:284-94. doi: 10.1097/NEN.0000000000 000032.

6. Zampieri S, Pietrangelo L, Loefler S, et al. Lifelong Physical Exercise Delays AgeAssociated Skeletal Muscle Decline. J Gerontol A Biol Sci Med Sci 2015;70:163-73. doi: 10.1093/gerona/glu006. Epub 2014 Feb 18.

7. Kern H, Pelosi L, Coletto L, et al. Atrophy/hypertrophy cell signaling in muscles of young athletes trained with vibrationalproprioceptive stimulation. Neurol Res. 2011;33:998-1009.

8. Kern H. Funktionelle Elektrostimulation Paraplegischer Patienten. ÖZPM, Österreichi sche 


\section{Biology of muscle atrophy and of its recovery by FES}

Eur J Transl Myol - Basic Appl Myol 2015; 25 (4): 221-230

Zeitschrift für Physikalische Medizin 1995;5:1-75. ISSN 1021-4348.

9. Kern H, Boncompagni S, Rossini K, et al. Longterm denervation in humans causes degeneration of both contractile and excitation contraction coupling apparatus, which is reversible by functional electrical stimulation (FES). A role for myofiber regeneration? J Neuropathol Exp Neurol 2004;63:919-31.

10. Kern H, Rossini K, Carraro U, et al. Muscle biopsies show that FES of denervated muscles reverses human muscle degeneration from permanent spinal motoneuron lesion. J Rehabil Res Dev 2005;42:43-53.

11. Boncompagni S, Kern $\mathrm{H}$, Rossini $\mathrm{K}$, et al. Structural differentiation of skeletal muscle fibers in the absence of innervation in humans. Proc Natl Acad Sci USA 2007;104:19339-44.

12. Kern H, Hofer C, Mayr W. Protocols for clinical work package of the European project RISE. Eur J Transl Myol/ Basic Appl Myol 2008;18:39-44.

13. Kern H, Carraro U, Adami N, et al. One year of home-based Functional Electrical Stimulation (FES) in complete lower motor neuron paraplegia: Recovery of tetanic contractility drives the structural improvements of denervated muscle. Neurol Res 2010;32:5-12,doi: 10.1189/ 184313209 X385644.

14. Kern H, Carraro U, Adami N, et al. Home-based functional electrical stimulation rescues permanently denervated muscles in paraplegic patients with complete lower motor neuron lesion. Neurorehabil Neural Repair 2010;24:709-21. doi: 10.1177/ 1545968310366129. Epub 2010 May 11.

15. Rossini K, Zanin ME, Carraro U. To stage and quantify regenerative myogenesis in human longterm permanent denervated muscle. Basic Appl Myol 2002;12:277-87.

16. Carraro U, Rossini K, Mayr W, Kern H. Muscle fiber regeneration in human permanent lower motoneuron denervation: relevance to safety and effectiveness of FES-training, which induces muscle recovery in SCI subjects. Artif Organs 2005;29:187-91.

17. Carraro U, Boncompagni S, Gobbo V, et al. Persistent muscle fiber regeneration in long term denervation. Past, present, future. Eur J Transl Myol 2015;25:77-92.
18. Gargiulo P, Helgason $\mathrm{T}$, Reynisson PJ, et al. Monitoring of muscle and bone recovery in spinal cord injury patients treated with electrical stimulation using three-dimensional imaging and segmentation techniques: methodological assessment. Artif Organs 2011:35:275-81. doi: 10.1111/j.1525-1594.2011.01214.x.

19. Gargiulo P, Reynisson PJ, Helgason B, et al. Muscle, tendons, and bone: structural changes during denervation and FES treatment. Neurol Res 2011;Sep:33(7):750-8. doi: 10.1179/174313 2811Y.0000000007.

20. Carraro U, Edmunds KJ, Gargiulo P. 3D false color computed tomography for diagnosis and follow-up of permanent denervated human muscles submitted to home-based Functional Electrical Stimulation. Eur J Transl Myol - Basic Appl Myol 2015;25:129-40.

21. Wang R, Meinel FG, Schoepf UJ, Canstein C, Spearman JV, De Cecco CN. Performance of Automated Software in the Assessment of Segmental Left Ventricular Function in Cardiac CT: Comparison with Cardiac Magnetic Resonance. Eur Radiol. 2015 Apr 30. [Epub ahead of print]

22. Schils SJ, Turner TA. Functional Electrical Stimulation for equine epaxial muscle spasms:retrospective study of 241 clinical cases. Comparative Exercise Physiology 2014;10:89-97.

23. Ravara B, Gobbo V, Carraro U et al. Functional electrical stimulation as a safe and effective treatment for equine epaxial muscle spasms: Clinical evaluations and histochemical morphometry of mitochondria in muscle biopsies. Eur J Transl Myol - Basic Appl Myol 2015; 25 (2): 109-120.

24. Mosole S, Zampieri S, Germinario E, et al. Structural and functional characteristics of denervated muscles from oldest-old rats: a relevant animal model for FES of denervated myofibers of the diaphragm in ALS? Eur J Transl Myol/Basic Appl Myol 2015; 25: 151.

25. Donà $\mathrm{M}$, Sandri $\mathrm{M}$, Rossini $\mathrm{K}$, Dell'Aica I, Podhorska-Okolow M, Carraro U. Functional in vivo gene transfer into the myofibers of adult skeletal muscle. Biochem Biophys Res Commun. 2003;312:1132-8.

26. Taylor JA, Babbs CF, Alzghoul MB, et al. Optimization of Ectopic Gene Expression in 


\section{Biology of muscle atrophy and of its recovery by FES}

Eur J Transl Myol - Basic Appl Myol 2015; 25 (4): 221-230

Skeletal Muscle through DNA Transfer by Electroporation. BMC Biotechnology 2004;4:11.

27. Mammucari C, Gherardi G, Zamparo I, et al. The mitochondrial calcium uniporter controls skeletal muscle trophism in vivo. Cell Rep. 2015 Mar 3;10(8):1269-79. doi:10.1016/j.celrep. 2015.01. 056. Epub 2015 Feb 26.
28. Kern H, Barberi L, Löfler S, et al. Electrical stimulation counteracts muscle decline in seniors. Front Aging Neurosci 2014;Jul 24:6:189. doi: 10.3389/fnagi.2014.00189. eCollection 2014.

29. Kern H. Electrical Stimulation on Paraplegic Patients. Eur J Trans Myol/ Basic Appl Myol 2014;24:75-157. 